\title{
Comments on "The severity of non-alcoholic fatty liver disease is associated with gut dysbiosis and shift in the metabolic function of the gut microbiota"
}

\author{
Anna Egresi, Krisztina Hagymási, Gabriella Lengyel \\ The 2nd Department of Internal Medicine, Semmelweis University, H-1088 Budapest, Hungary
}

Correspondence to: Dr. Gabriella Lengyel, the 2nd Department of Internal Medicine, Semmelweis University, Szentkirályi Street 46, H-1088 Budapest, Hungary. E-mail: lengyel.gabriella@med.semmelweis-univ.hu

\begin{abstract}
How to cite this article: Egresi A, Hagymási K, Lengyel G. Comments on "The severity of non-alcoholic fatty liver disease is associated with gut dysbiosis and shift in the metabolic function of the gut microbiota". Hepatoma Res 2016;2:328-30.
\end{abstract}

Article history: Received: 20-07-2016 Accepted: 25-11-2016 Published: 13-12-2016

"Boursier J, Mueller O, Barret M, Machado M, Fizanne L, Araujo-Perez F, Guy CD, Seed PC, Rawls JF, David LA, Hunault G, Oberti F, Calès P, Diehl $A M$. The severity of nonalcoholic fatty liver disease is associated with gut dysbiosis and shift in the metabolic function of the gut microbiota. Hepatology 2016;63:764-75."

Non-alcoholic fatty liver disease (NAFLD) has a rising prevalence worldwide. It is characterized with lipid deposition in hepatocytes that is unrelated to alcohol consumption. Insulin resistance and oxidative damage plays a key role in its pathogenesis. ${ }^{[1]}$ NAFLD is a complex disease, classified in simple steatosis (SS) and non-alcoholic steatohepatitis (NASH). Lifestyle changes and treatment of hyperinsulinaemia could reverse SS. However, $20-30 \%$ of NAFLD patients develop to NASH, which could lead to liver fibrosis, cirrhosis and cancer. ${ }^{[2]}$

Recently, the intestinal microbial flora has gained great attention in various diseases, such as obesity, metabolic syndrome, diabetes, and cardiovascular diseases. ${ }^{[3]}$ Gut dysbiosis, especially the microbial translocation and their products such as endotoxin (lipopolysaccharides) across the intestinal gut barrier is highly investigated in patients with chronic liver diseases. ${ }^{[4]}$ Besides that, gut microbiota may influence the pathogenesis of NAFLD by increased production and absorption of gut short-chain fatty acids; changes in dietary choline metabolism; altered bile acid pools; increased production of microbiotaderived endogenous ethanol; and interaction between dietary factors and microbiota. ${ }^{[5]}$ Nowadays there is no evidence-based, effective therapy of NAFLD. Current therapy for NAFLD includes lifestyle interventions, medical treatment (e.g. antioxidants, oral hypoglycaemic agents, and lipid-lowering agents), and bariatric surgery. Lately, probiotics have been discussed as a potential treatment of NAFLD. ${ }^{[6]}$

Boursier et al. ${ }^{[7]}$ present a remarkable study about the analysis of composition of gut microbiota of stool samples from patients affected by this disease.

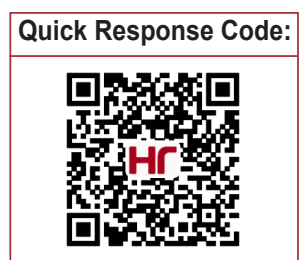


They used $16 \mathrm{~S}$ ribosomal RNA gene sequencing to determine the microbial flora of biopsy-proven NASH patients. Here, we highlight the importance of this work, because of its novelty in the demonstration of the association between gut dysbiosis and severity of the disease. Another great advantage of this study is the well phenotyped liver lesions of the studied group with diagnostic liver biopsy. In so far, the histological assessment of liver samples is the gold standard of chronic liver diseases, which is an invasive intervention. Not only NAFLD, but NASH can be hardly diagnosed with clinical, laboratory parameters. For this reason there is a great claim worldwide for easy, reproducible, cheap, safe, non-invasive scores or imaging modalities to identify NAFLD or NASH. ${ }^{[8-11]}$

In particular, the authors reported an increased abundance of Bacteroides genus was independently associated with NASH and Ruminococcus abundance was independently associated with fibrosis severity (> F2). Besides, patients with NASH had lower abundance of Prevotella. ${ }^{[7]}$ The exact mechanism of the liver injury is not yet clear. However, metabolites of the members of the Bacteroides family directly stimulate the farnesoid $X$ receptor (FXR), a ligandactivated transcription factor, which plays an important role in the pathogenesis of liver damage. They improve the obesity phenotype including body weight gain, liver damage, lipid metabolism in a mouse model. ${ }^{[12]}$ Despite the relatively small number of cases $(n=57)$, these results show that analysis of gut microbiota is a good tool to gain information about NAFLD severity. According to this work, dysbiosis may have a significant role in the pathogenesis of human NAFLD/NASH. Moreover gut microbiota analysis adds prognostic information to NAFLD severity. ${ }^{[7]}$

Recent studies show, that probiotic/prebiotic supplementation may be useful in both animal and human models. Bifidobacterium and Lactobacillus strains are the most widely used bacteria. ${ }^{[13]}$ Control of the bacterial flora lowers proinflammatory cytokine production (tumor necrosis factor- $\alpha$, interleukin-6, interferon- $\gamma$ ) via down-regulation of the nuclear factor kappa B, and decreases the oxidative stress. Probiotic can reduce the urease activity of bacterial microflora, decreases fecal $\mathrm{pH}$ value and reduces amino-acid fermentation and ammonia adsorption. They may reduce aminotransferases (alanine aminotransferase, aspartate transaminase), improve the lipid status (total cholesterol, low-density lipoprotein) in NAFLD patients. ${ }^{[6,14-17]}$ In fact, probiotic was reported to improve liver histology, and reduce hepatic total fatty acid content in an animal model of NAFLD. In addition probiotic therapy with lifestyle modification significantly decreased fibrosis scores, as determined by transient elastography compared with placebo. ${ }^{[18]}$ In fact, it can improve disease severity. Probiotics are consumed in various forms, such as fermented foods, like yogurt, cheese and other fermented milk products. The oral intake of probiotics is recommendable for the prevention and treatment of obesity, insulin resistance, type 2 diabetes and NAFLD. As a co-adjuvant therapy, probiotic combination with metformin can lower liver aminotransferases better than metformin alone in patients with NASH. ${ }^{[19,20]}$ The combination of cholesterol-lowering probiotics and anthraquinone increase the therapeutic effect on NAFLD by affecting the process of fat metabolism in rats (up-regulation of CYP7A1, LDL-R, FXR mRNA, PPAR- $\alpha$ protein and down-regulation of HMGCR, PPAR-y and SREBP-1c). ${ }^{[21]}$

These findings confirm that dietary interventions can affect the composition and diversity of gut microbiota.

Finally, association of gut dysbiosis with histological subtypes of NAFLD may be a prognostic factor of liverrelated morbidity and mortality. Analysis of microbial composition may be a useful predictor of disease stage. However additional analysis will have to explain how metabolic functions of the gut microbiota might have role in $\mathrm{NASH}$ pathogenesis and progression. Further studies are required to understand the precise mechanism of how gut microbiota affect the pathomechanism of NAFLD/NASH and the role of probiotics in the therapy of the disease. Since there is no available treatment for NAFLD/NASH yet, probiotic therapy, as a safe, inexpensive and noninvasive strategy, may be a good alternative to reduce pathophysiological symptoms and improve different types of liver diseases without side effects.

\section{Financial support and sponsorship}

None.

\section{Conflicts of interest}

There are no conflicts of interest.

\section{Patient consent}

There is no patient involved.

\section{Ethics approval}

Ethics approval is waived for this kind of article.

\section{REFERENCES}

1. Filozof C, Goldstein BJ, Williams RN, Sanyal A. Non-alcoholic steatohepatitis: limited available treatment options but promising drugs in development and recent progress towards a regulatory approval pathway. Drugs 2015;75:1373-92. 
2. Singh S, Allen AM, Wang Z, Prokop LJ, Murad MH, Loomba R. Fibrosis progression in nonalcoholic fatty liver vs nonalcoholic steatohepatitis: a systematic review and meta-analysis of paired-biopsy studies. Clin Gastroenterol Hepatol 2015;13:643-54.e1-9; quiz e39-40.

3. Boursier J, Diehl AM. Nonalcoholic fatty liver disease and the gut microbiome. Clin Liver Dis 2016;20:263-75.

4. Xie G, Wang X, Liu P, Wei R, Chen W, Rajani C, Hernandez BY, Alegado R, Dong B, Li D, Jia W. Distinctly altered gut microbiota in the progression of liver disease. Oncotarget 2016;7:19355-66.

5. Yu J, Marsh S, Hu J, Feng W, Wu C. The pathogenesis of nonalcoholic fatty liver disease: interplay between diet, gut microbiota, and genetic background. Gastroenterol Res Pract 2016;2016:2862173.

6. Nabavi S, Rafraf M, Somi MH, Homayouni-Rad A, AsghariJafarabadi M. Effects of probiotic yogurt consumption on metabolic factors in individuals with nonalcoholic fatty liver disease. J Dairy Sci 2014;97:7386-93.

7. Boursier J, Mueller O, Barret M, Machado M, Fizanne L, AraujoPerez F, Guy CD, Seed PC, Rawls JF, David LA, Hunault G, Oberti F, Calès P, Diehl AM. The severity of nonalcoholic fatty liver disease is associated with gut dysbiosis and shift in the metabolic function of the gut microbiota. Hepatology 2016;63:764-75.

8. Dvorak K, Stritesky J, Petrtyl J, Vitek L, Sroubkova R, Lenicek M, Smid V, Haluzik M, Bruha R. Use of non-invasive parameters of non-alcoholic steatohepatitis and liver fibrosis in daily practice - an exploratory case-control study. PLoS One 2014;9:e111551.

9. Ferraioli G, Tinelli C, Lissandrin R, Zicchetti M, Dal Bello B, Filice G, Filice C. Controlled attenuation parameter for evaluating liver steatosis in chronic viral hepatitis. World J Gastroenterol 2014;20:6626-31.

10. Fitzpatrick E, Dhawan A. Noninvasive biomarkers in non-alcoholic fatty liver disease: current status and a glimpse of the future. World $J$ Gastroenterol 2014;20:10851-63.

11. Nalbantoglu IL, Brunt EM. Role of liver biopsy in nonalcoholic fatty liver disease. World J Gastroenterol 2014;20:9026-37.
12. Zhang X, Osaka T, Tsuneda S. Bacterial metabolites directly modulate farnesoid X receptor activity. Nutr Metab (Lond) 2015;12:48.

13. Paolella G, Mandato C, Pierri L, Poeta M, Di Stasi M, Vajro P. Gutliver axis and probiotics: their role in non-alcoholic fatty liver disease. World J Gastroenterol 2014;20:15518-31.

14. Haque TR, Barritt ASt. Intestinal microbiota in liver disease. Best Pract Res Clin Gastroenterol 2016;30:133-42.

15. Dugan CE, Aguilar D, Park YK, Lee JY, Fernandez ML. Dairy consumption lowers systemic inflammation and liver enzymes in typically low-dairy consumers with clinical characteristics of metabolic syndrome. J Am Coll Nutr 2016;35:255-61.

16. Imani Fooladi AA, Mahmoodzadeh Hosseini H, Nourani MR, Khani S, Alavian SM: Probiotic as a novel treatment strategy against liver disease. Hepat Mon 2013;13:e7521.

17. Liu JE, Zhang Y, Zhang J, Dong PL, Chen M, Duan ZP. Probiotic yogurt effects on intestinal flora of patients with chronic liver disease. Nurs Res 2010;59:426-32.

18. Takahashi Y, Sugimoto K, Inui H, Fukusato T. Current pharmacological therapies for nonalcoholic fatty liver disease/ nonalcoholic steatohepatitis. World J Gastroenterol 2015;21:3777-85.

19. Saez-Lara MJ, Robles-Sanchez C, Ruiz-Ojeda FJ, Plaza-Diaz J, Gil A. Effects of probiotics and synbiotics on obesity, insulin resistance syndrome, type 2 diabetes and non-alcoholic fatty liver disease: a review of human clinical trials. Int J Mol Sci 2016;17:E928.

20. Shavakhi A, Minakari M, Firouzian H, Assali R, Hekmatdoost A, Ferns G. Effect of a probiotic and metformin on liver aminotransferases in non-alcoholic steatohepatitis: a double blind randomized clinical trial. Int J Prev Med 2013;4:531-7.

21. Mei L, Tang Y, Li M, Yang P, Liu Z, Yuan J, Zheng P. Coadministration of cholesterol-lowering probiotics and anthraquinone from Cassia obtusifolia L. ameliorate non-alcoholic fatty liver. PLoS One 2015; 10:e0138078. 